
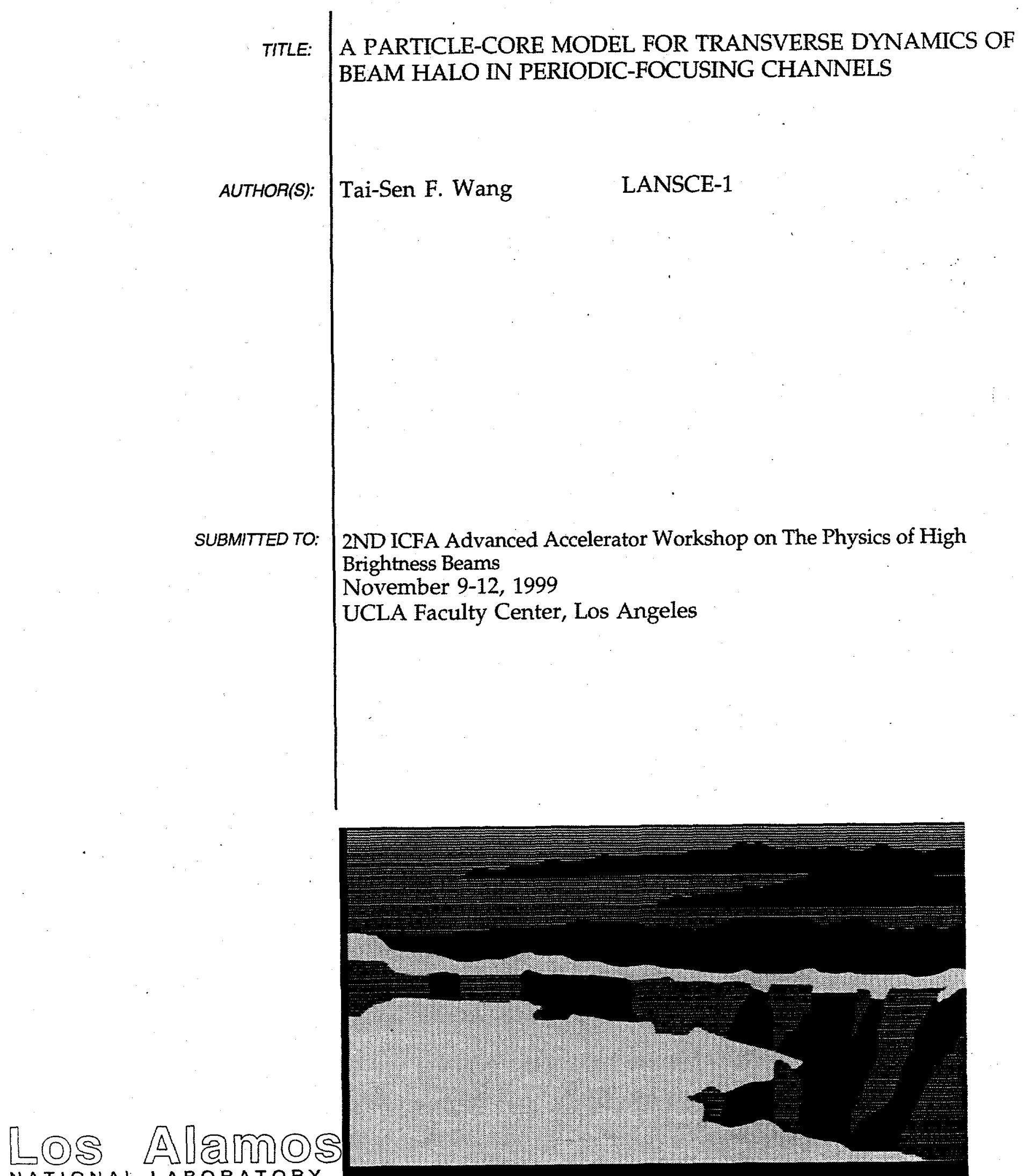

NATIONAL LABORATORY

Los Alamos National Laboratory, an affirmative action/equal opportunity employer, is operated by the University of Califomia for the U.S. Department of Energy Los Alamos National Laboratory, an aftimative action under contract W-7405-ENG-36. By acceptance of this article, the publisher recognizes that the U.S. Govemment retains a non Alamos National Laboratory requests that the publisher identify this article as work performed under the auspices of the U.S. Department of Energy. 


\section{DISCLAIMER}

This report was prepared as an account of work sponsored by an agency of the United States Government. Neither the United States Government nor any agency thereof, nor any of their employees, make any warranty, express or implied, or assumes any legal liability or responsibility for the accuracy, completeness, or usefulness of any information, apparatus, product, or process disclosed, or represents that its use would not infringe privately owned rights. Reference herein to any specific commercial product, process, or service by trade name, trademark, manufacturer, or otherwise does not necessarily constitute or imply its endorsement, recommendation, or favoring by the United States Government or any agency thereof. The views and opinions of authors expressed herein do not necessarily state or reflect those of the United States Government or any agency thereof. 


\section{DISCLAIMER}

Portions of this document may be illegible in electronic image products. Images are produced from the best available original document. 


\title{
A PARTICLE-CORE MODEL FOR TRANSVERSE DYNAMICS OF BEAM HALO IN PERIODIC-FOCUSING CHANNELS
}

\author{
TAI-SEN F. WANG \\ MS H808, Los Alamos National Laboratory \\ Los Alamos, New Mexico 87545, USA \\ E-mail: twang@lanl.gov
}

\begin{abstract}
This work is a numerical study of the particle dynamics in the halo region of mismatched charged-particle beams propagating through periodic-focusing channels using a recently developed technique based on the particle-core model. Numerical examples and preliminary results of finding the boundaries of the resonant region in the phase space where particles can be driven into the beam halo will be presented.
\end{abstract}

\section{Introduction}

In recent years, there has been a growing of interest in the halo formation in intense ion beams, and progress has been made in understanding the particle dynamics in the beam halo by using both analytical approach and numerical simulations. ${ }^{1-16}$ In the analytical studies, the particle-core model has provided insight of halo formation for a mismatched beam propagating through an axisymmetric uniform-focusing channel. However, the progress in applying the same model to a beam propagating through a periodic-focusing channel remains limited. ${ }^{13-16}$ The main obstacle stems from the flutter in the beam envelope and in the particle orbit introduced by the focusing. Owing to this flutter, the dimension of a Poincaré section made by strobing a particle's phase space is usually higher than two. ${ }^{17}$ One then has to slice the phase space or to project the higher-dimensional Poincaré "plot" onto a two-dimensional plane in order to study the particle dynamics. The former approach is complicated by the searching for suitable phase-space slices, while the latter approach may result in a plot with few features to be deciphered.

Recently, a method has been developed to show that by using proper canonical transformations and strobing, the flutter due to the periodic focusing can be minimized in the new phase-space variables so that the primary resonance between the particle and the core can be manifested in the projected stroboscopic plots. ${ }^{15,16}$ Using this method, it was found that the parametric resonance, like the one studied in the uniform-focusing case, is still the major mechanism to cause the large-amplitude oscillation of halo particles. We also learned that certain particles with initial oscillation amplitudes slightly larger than the core radius, but not in the halo region, can be brought into resonance 
with the core oscillation by the fluctuation of the periodic focusing. Since the transverse density profile of a realistic beam inevitably has some tails instead of a sharp-edged distribution, some particles in the tails of a mismatched beam can be driven into halo by this kind of mechanism. Previous particle-core studies of halo formation in the uniform-focusing channel found the existence of a separatrix between the core ard the resonant region, and concluded that either the halo particles were initially in the resonant region ${ }^{5,12}$ or the halo particles were brought across the separatrix from the core by some kind of process like coherent instability. ${ }^{18}$ For periodic-focusing channels, the flutter caused by the periodic focusing introduces another possible process of halo formation.

This present work is intended to be a continuation of that in Ref. 16 . The main purpose here is to present some numerical results not covered in the earlier papers ${ }^{15,16}$ including the preliminary results from searching for the approximated boundaries in the phase space for halo formation. To make this paper somewhat self-contained, we will first review the proposed method for studying the halo dynamics in periodic focusing channels before giving the numerical results. We will omit discussion of chaotic motion and stability of the core oscillation.

\section{The Model and the Approach}

The system considered here has a test particle and a continuous beam (the core) propagating in a periodic-focusing channel with a speed $v$ in the axial direction, the $z$ direction. Particles are focused in the transverse direction by a linear force that varies in the $z$ direction according to $G F(k z)$, where $G$ is the maximal gradient of the focusing (or defocusing) strength, $F(k z)$ is a periodic function of $z, k$ is the wave number of the periodicity, and the maximum of $|F(k z)|$ is normalized to unity. The particles in the core are assumed to follow the Kapchinskij and Vladimirskij (KV) ${ }^{19}$ distribution in transverse phase space. In the following, we shall consider the axisymmetricand the quadrupole-focusing separately.

\subsection{Axisymmetric-Focusing Systems}

In this case, the dimensionless equations for the beam envelope and the transverse motion of the test particle are

$$
\frac{d^{2} X}{d \tau^{2}}+Q^{2} X F(r)-\frac{\eta}{X}-\frac{1}{X^{3}}=0
$$

and 


$$
\frac{d^{2} x}{d \tau^{2}}-\frac{L^{2}}{x^{3}}+Q^{2} x F(\tau)= \begin{cases}\eta x / X^{2}, & \text { for } x \leq X \\ \eta / x, & \text { for } x>X\end{cases}
$$

respectively, where $X=X_{r} \sqrt{k / \epsilon}, x=x_{r} \sqrt{k / \epsilon}, \tau=k z, X_{r}$ is the beam envelope, $x_{r}$ is the transverse displacement of the particle from the symmetry axis of the system, $\epsilon$ is the beam emittance, $L=L_{r} /\left(m_{0} \gamma v \epsilon\right), \eta=$ $q I /\left(2 \pi \epsilon_{0} m_{0} \gamma^{3} v^{3} k \epsilon\right), Q^{2}=q G /\left(m_{0} \gamma v^{2} k^{2}\right), q$ and $m_{0}$ are the charge and the rest mass of a beam particle, respectively, $\gamma$ is the relativistic mass factor, $I$ is the beam current, $\epsilon_{0}$ is the permittivity of free space, and $L_{r}$ is the angular momentum of the test particle about the $z$ axis.

The technique employed in Refs. 15 and 16 for reducing the flutter is to use new variables $\left(u_{e}, w_{e}\right)$ and $(u, w)$ which are related to $X_{e}, d X_{e} / d \tau, x$, and $d x / d \dot{\tau}$ via the canonical transformations

$$
\left(u_{e}, w_{e}\right)=\left(\frac{X}{X_{m}}, \frac{d u_{e}}{d s}\right)=\left(\frac{X}{X_{m}}, X_{m} \frac{d X}{d \tau}-X \frac{d X_{m}}{d \tau}\right)
$$

and

$$
(u, w)=\left(\frac{x}{X}, \frac{d u}{d \psi}\right)=\left(\frac{x}{X}, X \frac{d x}{d \tau}-x \frac{d X}{d \tau}\right),
$$

where $X_{m}(\tau)=X_{m}(\tau+2 \pi)$ is the envelope of the matched core; the new times $s$ and $\psi$ are defined by $d s=d \tau / X_{m}^{2}$ and $d \psi=d \tau / X^{2}$. In terms of the new variables, Eqs. (1) and (2) can be rewritten as

$$
\begin{gathered}
\frac{d u_{e}}{d s}=w_{e} \\
\frac{d w_{e}}{d s}=\frac{1}{u_{e}^{3}}-u_{e}+\eta X_{m}^{2}\left(\frac{1}{u_{e}}-u_{e}\right), \\
\frac{d u}{d \psi}=w
\end{gathered}
$$

and

$$
\frac{d w}{d \psi}=\frac{L^{2}}{u^{3}}-u+\eta X^{2} \Theta(u-1)\left(\frac{1}{u}-u\right),
$$

where $\Theta(x)$ is the Heaviside step function. We notice that the focusing function $F$ is eliminated in Eqs. (5) to (8). Furthermore, the flutter due to the periodic focusing which is only a fraction of $X_{m}$ or $X$ is now contained in the terms proportional to the beam current. Therefore in the integrated solutions, the flutter in the new phase-space variables is substantially reduced. When $I=0$, the flutter in these new variables is suppressed completely. We also note that 
one can also choose to "normalize" $\tau$ and $x$ by $X_{m}$ instead of $X$; the choice made here is solely for simplicity. In terms of the variables $u$ and $w$, one finds that the Hamiltonian corresponding to Eqs. (7) and (8) is a constant for particles inside the phase-space ellipse of the beam core, so that particles in the core will remain inside the core. For particles outside the core ellipse, the Hamiltonian is time dependent and non-integrable except for some special cases.

The proposed approach is to study the dynamics of beam halo in the phase space of $(u, w)$. Since the oscillations in $u$ and $u_{e}$ are not periodic in most cases, stroboscopic plots are made by strobing at a fixed value of $u_{e}$ or $w_{e}$ (e.g., at the local maxima where $w_{e}=0$ ), instead of fixed time period, to minimize the phase shifting between the strobing and the core oscillation. Numerical results indicate that strobing at a constant period does create a larger spread of points mostly due to the phase shift between the strobing and the envelope oscillation (see Figs. 2 and 3 below). Thus, the stroboscopic plots proposed here are different from the usual Poincaré plots (plots made from two-dimensional Poincaré maps). These two kinds of plots are the same for uniform-focusing channels where $u_{e}$ is periodic. We shall loosely call the relation that links one snapshot to the next the "stroboscopic map". At very high beam current, particle motion can become chaotic. One of the deficiencies in projecting a higher-dimension phase-space section onto a two-dimensional plane is the difficulty in detecting the onset of chaotic motion. Another deficiency is that discerning higher-order resonances can be hard or impossible most of the time.

\subsection{Quadrupole-Focusing Systems}

For quadrupole-focusing systems, the equations for the beam envelope and particle motion in the $x$-direction are:

$$
\frac{d^{2} X}{d \tau^{2}}+Q^{2} X F(\tau)-\frac{2 \hat{\eta}}{X+Y}-\frac{\epsilon_{x}^{2}}{X^{3}}=0
$$

and

$$
\frac{d^{2} x}{d \tau^{2}}+Q^{2} x F(\tau)-\frac{2 \hat{\eta} x}{\Xi_{x}\left(\Xi_{x}+\Xi_{y}\right)}=0
$$

respectively, where $X=X_{r} \sqrt{k}, Y=Y_{r} \sqrt{k}, X_{r}$ and $Y_{r}$ are the beam envelopes in the $x$ and $y$ directions, respectively, $x=x_{r} \sqrt{k}, x_{r}$ is the displacement of the beam particle in the $x$ direction from the beam axis, $\hat{\eta}=q I /\left(2 \pi \epsilon_{0} m_{0} \gamma^{3} v^{3} k\right)$, $\epsilon_{x}$ is the beam emittance in the $x$ direction, $\Xi_{x}=\left(X^{2}+\xi\right)^{1 / 2}$, and $\Xi_{y}=$ $\left(Y^{2}+\xi\right)^{1 / 2}$. The value of $\xi$ is zero when the particle is inside the beam, and is given by the solution of the equation $\left(x / \Xi_{x}\right)^{2}+\left(y / \Xi_{y}\right)^{2}=1$ when the particle 
is outside the beam, where $y=y_{r} \sqrt{k}$, and $y_{r}$ is the excursion of the beam particle in the $y$ direction from the beam axis. The equations for the beam envelope and particle motion in the $y$ direction are similar.

The method proposed for axisymmetric systems can be generalized to quadrupole-focusing systems by the change of variables according to $u_{x}=x / X$, $w_{x}=X(d x / d \tau)-x(d X / d \tau), u_{e x}=X_{m} / X, w_{e x}=X\left(d X_{m} / d \tau\right)-X_{m}(d X / d \tau)$, and similar change of variables of $y$ direction. However, due to a lack of good guidance to the global phase-space structure of this kind of dynamic system, attempts in making two-dimensional plots have only very limited success. It is found that for a particle having nonzero angular momentum, even a small amount, the points on the plot are totally dispersed. It appears that twodimensional plots, except for some special cases, do not seem to provide insightful displays of the global system behavior. Thus, to deal with a problem of two degrees of freedom like this, it is necessary to consider the $x$ and $y$ motion of the particle separately by setting one of the coordinates to zero, e.g., $y=0$ and $d y / d \tau=0$.

\section{Numerical Results}

Figures 1 and 2 show examples of stroboscopic plots made by strobing at the local minima of $u_{e}$ for $F(\tau)=\cos ^{2} \tau$. The parameter values considered are $L=0, Q^{2}=0.1202, \eta=0.2082$, and $u_{e}(\tau=0)=0.8$. For a particle inside the matched core, the phase advance per period (or the tune) is about $90^{\circ}$ at zero current and $60^{\circ}$ at full beam current. These plots were created by first computing $x, d x / d \tau, X, d X / d \tau, X_{m}$, and $d X_{m} / d \tau$ simultaneously using Eqs. (1) and (2). The quantities $u$ and $w$ were then calculated using Eq. (4).

As reported in Ref. 16, that when tune depression is small, there are roughly five classes of particle motions. Class I particles remain inside the core. Class II particles are outside the phase-space ellipse of the core but not in resonance with the core oscillation because they experience less tune depression than the core particles. These particles stay close to the core. Particles in class III oscillate at frequencies near one-half the core oscillation frequency so they can resonate with the core motion to become halo particles. Class IV particles oscillate with large amplitudes and are depressed least in tune so they do not resonate with the core. Figure 1 shows a stroboscopic plot of four particles representing these four classes. The particles in this example start initially from rest with the values of $u$ equal to $0.6202,1.1410,1.5150$, and 3.3852 , for the class I, II, III, and IV particles, respectively. The points of the class II, III and IV particles appear to be scattered near the invariant curves of the Poincare plots for uniform-focusing channels. 


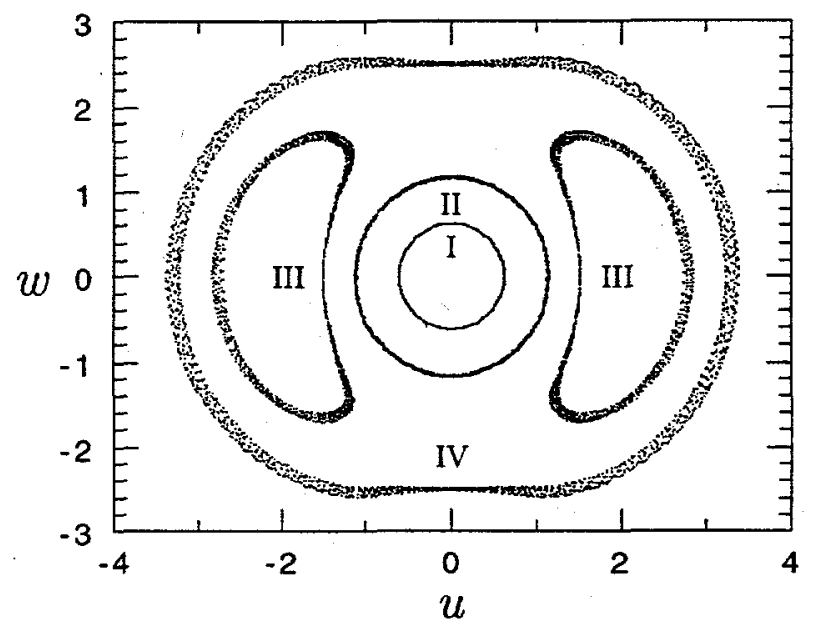

Figure 1: A stroboscopic plot on the $(u, w)$ phase plane showing four classes of particles discussed in the text.

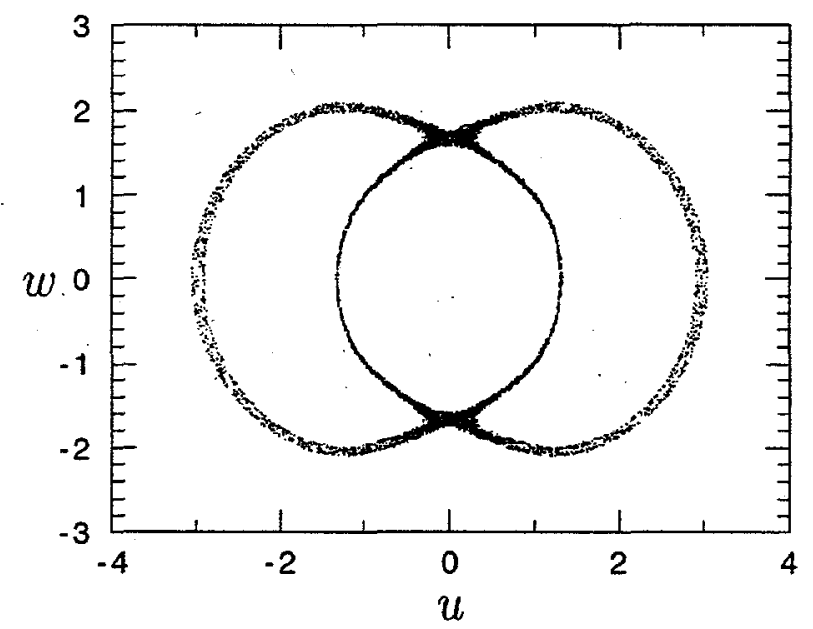

Figure 2: Stroboscopic plot for a class $V$ particle on the $(u, w)$ phase plane. 


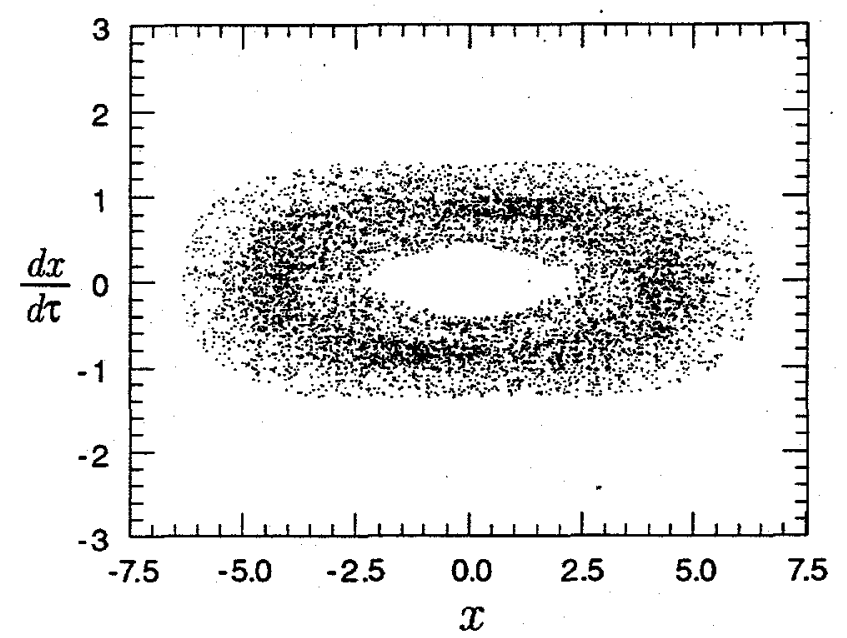

Figure 3: Shown is a plot on the $(x, d x / d \tau)$ phase plane made by a constant-period strobing for the same particle in Fig. 2.

The points of class V particles fall near the separatrices in a Poincare plot of the uniform-focusing case. Particles in this class can be driven into and out of resonance by the periodic-focusing and the flutter, an effect not found in the uniform-focusing systems and was left out by the smooth approximation in an earlier work. ${ }^{13,14}$ Since a realistic beam inevitably has some tails instead of a sharp-edged density profile in the transverse direction, in a mismatched beam, some of the particles in the tail may belong to classes III and V to become halo particles. Figure 2 is a stroboscopic plot for a class $\mathrm{V}$ particle with the initial condition $(u, w) \approx(1.3138,0)$. At large tune depression and strong focusing, the identification of particles' classes becomes ambiguous except for the class I particles.

To demonstrate the advantages of using the variables $(u, w)$ and the "stroboscopic plot", a plot on the $(x, d x / d \tau)$ phase plane made by strobing at the averaged envelope-oscillation period for the same particle in Fig. 2 is shown in Fig. 3, where we see that points are more scattered and the $2: 1$ resonance is indiscernible. The larger scattering of points in Fig. 3 is mainly due to the phase shift between the strobing and the envelope oscillation as well as the projection. It should be noted that although the stroboscopic plots of most particles on the $(u, w)$ phase plane do show spread points for most cases with nonzero beam current, special cases of very small spreads (or maybe zero spread) like 
the example shown in Fig. 4 do exist. The parameter values considered in Fig. 4 are $Q^{2}=0.2039, \eta=0.2765, u_{e}(0)=0.8, u(0)=1.4665 w(0)=0$, and $L=0$. For a particle inside the matched core, the phase advance per period (or the tune) is about $120^{\circ}$ at zero current and $80^{\circ}$ at full beam current. Here, the averaged phase advance of the particle is about $120.4385^{\circ}$ per period and is about one half of the averaged envelope phase advance per period. Without the guidance of an analytical solution, it is not clear if an invariant exists in this case.

Figure 5 shows an example of the stroboscopic plot for a particle with nonzero angular momentum, where $u_{e}(0)=1.6, u(0)=1.5614, w(0)=0$, $L^{2}=6, u=\sqrt{k / \epsilon}(r / X), r$ is the radial position of the particle in the polar coordinate that rotates with the same angular speed of the particle; the parameters of external focusing and beam current are the same as those considered in Fig. 4.

As mentioned earlier, the points of class $V$ particles fall near the separatrix in the phase space of a uniform-focused system. It is therefor interesting and useful to know the boundaries, if they exist, between the class II and the class V particles' phase-space areas. Numerical results from long-term particle tracking indicate that in the low tune depression regime, class II particles do stay near the core. This observation leads to the believe that a boundary separating the phase-space areas of the class II and the class V particles does exist when the tune depression is small. Figures 6 and 7 show the results of an attempt of searching for such kind of boundaries for zero angular momentum particles under the transverse focusing of $F(\tau)=\cos ^{2} \tau$. Figure 6 is for tune depressions of $120^{\circ}$ to $80^{\circ}$ and $100^{\circ}$; Fig. 7 is for tune depressions of $90^{\circ}$ to $50^{\circ}$ and $70^{\circ}$. Shown in these figures are the interceptions of the positive $u$ axis (where $w=0$ ) and the boundaries between the class II and the class V regions as functions of initial $u_{e}$. In both figures, the ranges of $u_{e}(0)$ is limited to $0.5 \leq u_{e}(0) \leq 0.98$ and $1.02 \leq u_{e}(0) \leq 1.6$. Since particle motion is sensitive to their initial conditions near the boundary, the results presented here should be considered as crude approximations. In the tracking, particles were all started at rest, i.e. $w=0$, and the boundaries were determined by the largest $u(0)$ for the motion of particles to remain in class II for a time duration of $\tau=1600$. As can be seen in the figures that the non-resonant region shrinks as the tune depression and mismatch increase. For $u_{e}(0)<0.9$ and $1.1<u_{e}(0)$, the boundaries are between $15 \%$ to $20 \%$ of the mismatched envelope in the higher tune depression cases $\left(\sigma / \sigma_{o}=80^{\circ} / 120^{\circ}\right.$ and $\left.50^{\circ} / 90^{\circ}\right)$, and are not very sensitive to the envelope mismatch. In the regions of $0.95<u_{e}(0)<1.05$ for $\sigma / \sigma_{o}=80^{\circ} / 120^{\circ}$, and $0.9<u_{e}(0)<1.1$ for $50^{\circ} / 90^{\circ}$, where the mismatch is small, the boundaries become less sensitive to the tune depression. 


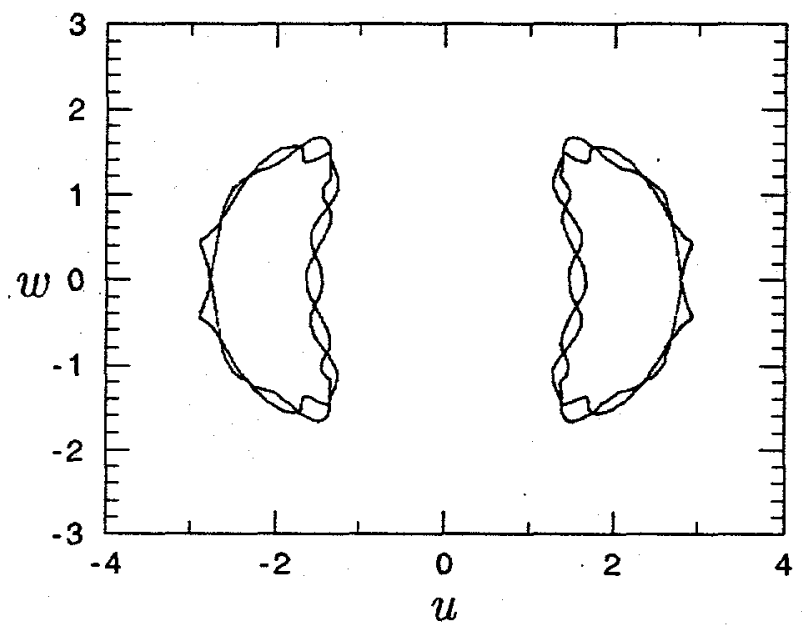

Figure 4: A stroboscopic plot on the $(u, w)$ phase plane showing very small spread of points. The parameter values are described in the text. The averaged phase advance of the particle is about $120.4385^{\circ}$ per period and is about one half of that of the envelope oscillation.

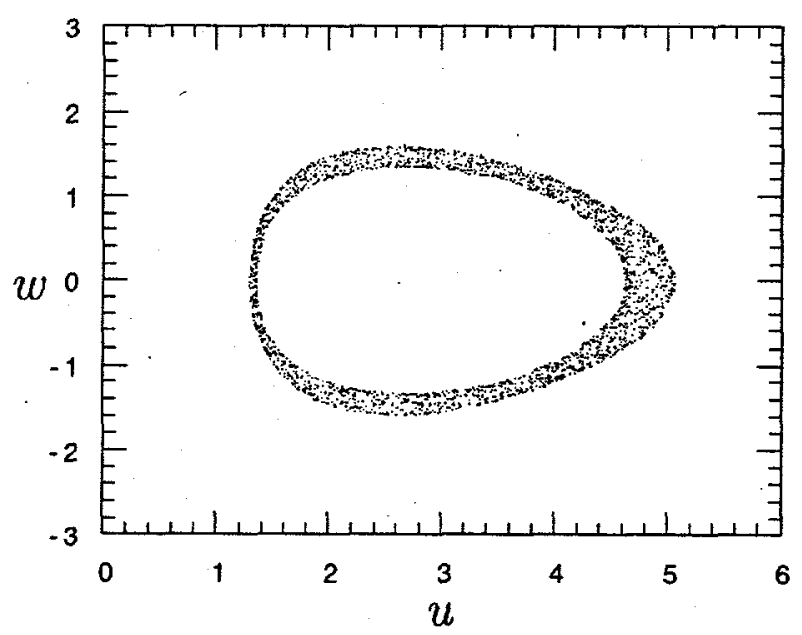

Figure 5: The stroboscopic plot for a particle with nonzero angular momentum. The parameter values are described in the text. 


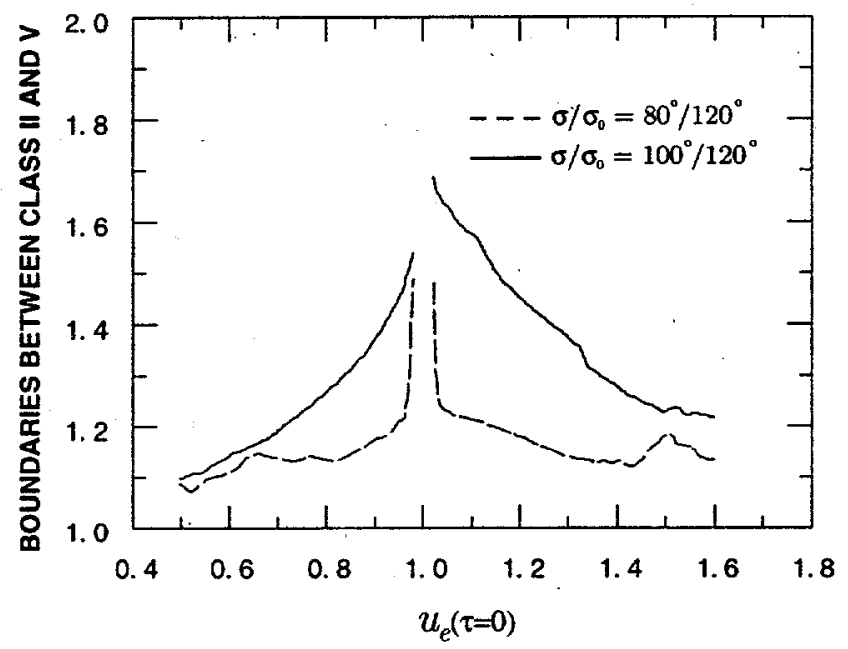

Figure 6: Shown are the interceptions of the positive $u$ axis (where $w=0$ ) and the boundaries between the class II and the class $\mathrm{V}$ regions as functions of initial $u_{e}$ for tune depressions of $120^{\circ}$ to $80^{\circ}$ (dashed curves) and $120^{\circ}$ to $100^{\circ}$ (solid curves). The transverse focusing considered is $F(\tau)=\cos ^{2} \tau$ and particles are assumed to have zero angular momentum.

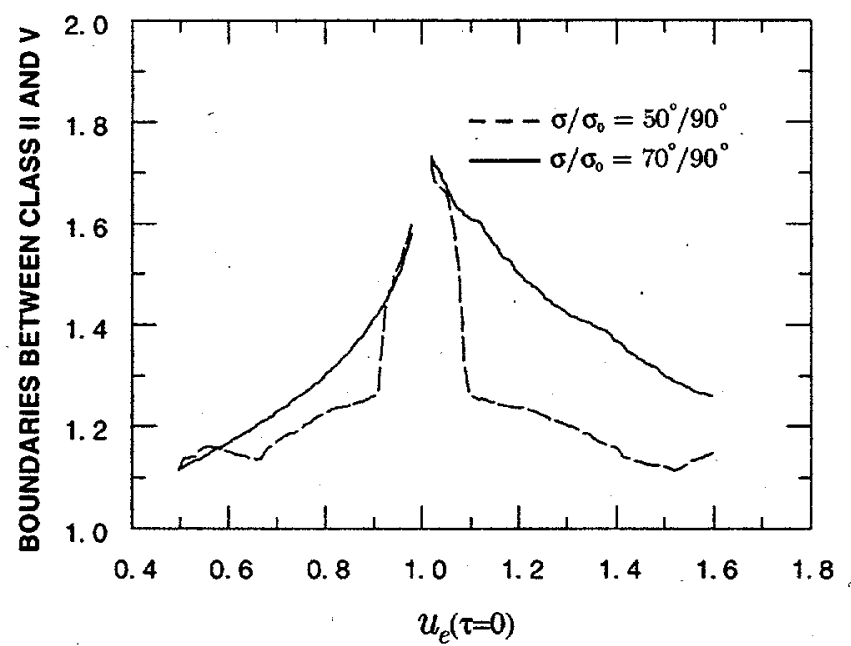

Figure 7: Shown are the interceptions of the positive $u$ axis (where $w=0$ ) and the boundaries between the class II and the class $\mathrm{V}$ regions as functions of initial $u_{e}$ for tune depressions of $90^{\circ}$ to $50^{\circ}$ (dashed curves) and $90^{\circ}$ to $70^{\circ}$ (solid curves). The transverse focusing considered is $F(\tau)=\cos ^{2} \tau$ and particles are assumed to have zero angular momentum. 


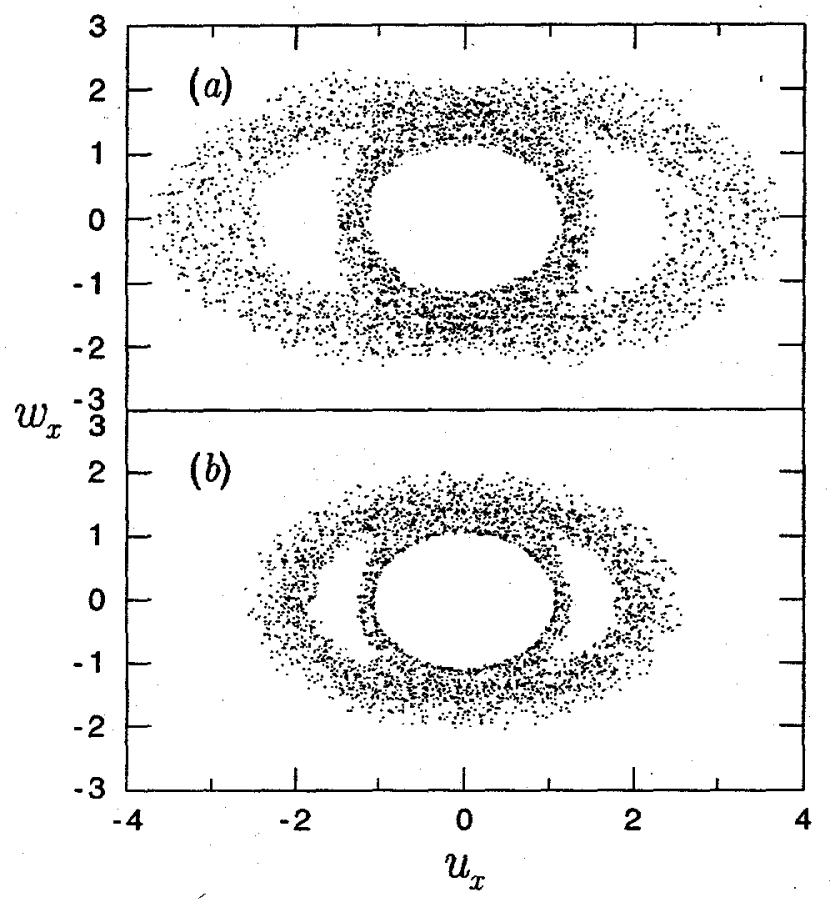

Figure 8: Stroboscopic plot showing the resonance of a halo particle with (a) the breathing mode, and (b) the quadrupole mode oscillations of the beam envelope in a quadrupolefocusing channel.

An example of applying the method to a quadrupole-focusing system is given in Fig. 8 where we considered the cases in which the envelope oscillations are close to one of the eigenmodes of the linearized envelope equations: the usual breathing mode with $X$ and $Y$ oscillating in phase, and the quadrupole mode with $X$ and $Y$ oscillating at $180^{\circ}$ off phase. The system parameters used in making the stroboscopic plots are $F(\tau)=\cos (\tau), \epsilon_{x}=\epsilon_{y}=1, Q^{2}=3.198$, and $\hat{\eta} / \epsilon=0.2502$. These parameter values correspond to a tune depression from $90^{\circ}$ to $70^{\circ}$ for particles inside the matched beam. The initial conditions used are $w_{e x}=w_{e y}=w_{x}=u_{y}=w_{y}=0$ and $u_{e x}=u_{e y}=0.8, u_{x} \approx 1.1958$, for (a); $u_{e x}=0.85, u_{e y}=1.15, u_{x} \approx 1.0844$ for (b). 


\section{Conclusions}

We have reviewed a recently developed method that employs the particle-core model for studying the dynamics of halo particles in a mismatched continuous beam propagating through a periodic-focusing channel. The study assumes that the beam-particle density and envelope are described by the KV distribution function and envelope equation. The method uses canonical transformations and a technique of strobing to reduce, in the new variables, the fluctuation due to the periodic focusing so that the dynamics of halo particles can be perceived through the stroboscopic plots. Using this method, it is found that the 2 to 1 parametric resonance, like the one studied in the uniform-focusing case, is still the major mechanism to cause the large-amplitude oscillation of halo particles. It is also found that certain particles with initial oscillation amplitudes slightly larger than the core radius, but not in the halo region, can be brought into resonance with the core oscillation by the fluctuation of the periodic focusing. Numerical examples were given for illustration and preliminary results of studying the boundaries of the resonant region in the phase space where particles can be driven into the beam halo were presented. The results show the decrease of non-resonant region in phase space as the tune depression and mismatch increase. It was also found that the boundary becomes less sensitive to the envelope mismatch when tune depression increases.

\section{Acknowledgments}

This work was supported by Los Alamos National Laboratory, under the auspices of the U.S. Department of Energy.

\section{References}

1. R. Jameson, Los Alamos National Laboratory Report LA-UR-93-1209 (1993).

2. J. S. O'Connell, T. P. Wangler, R. S. Mills, and K. R. Crandall, Proc. of 1993 Particle Accelerator Conf., p. 3657 (1993).

3. T. P. Wangler, Los Alamos National Laboratory Report LA-UR-94-1135 (1994).

4. J. Lagniel, Nucl. Instrum. Meth. A 345, 46 (1994); 345, 405 (1994).

5. R. Gluckstern, Phys. Rev. Lett. 73, 1247 (1994).

6. C. Chen, and R. C. Davidson, Phys. Rev. Lett. 72, 2195 (1994); Phys. Rev. E 49, 5679 (1994).

7. S. Y. Lee, and A. Riabko, Phys. Rev. E 51, 1609 (1995). 
8. A. Riabko, and S. Y. Lee, in Space Charge Dominated Beams and Applications of High Brightness Beams, AIP Conf. Proc. 377, p. 187 (1996).

9. C. Chen, and R. A. Jameson, Phys. Rev. E 52, 3074 (1995).

10. T. P. Wangler, R. W. Garnett, E. R. Gray, R. D. Ryne, and T.-S. Wang, Proc. of XVIII Intl. Linear Accelerator Conf., (CERN report CERN 96-07), p. 372 (1996).

11. H. Okamoto, and M. Ikegami, Phys. Rev. E 55, 4694 (1997).

12. T. P. Wangler, K. R. Crandall, R. Ryan, and T.-S. Wang, Phys. Rev. ST Accel. Beams 1, 084201 (1998).

13. M. Ikegami, and M. Mitzumoto, Proc. of XIX Intl. Linear Accelerator Conf., (Argonne National Laboratory Report No. ANL-98/28), p. 821 (1998).

14. M. Ikegami, Phys. Rev. E 59, 2330 (1999).

15. T. F. Wang and T. P. Wangler, in Proc. of 1999 Particle Accelerator Conf., IEEE Catalog No. 99CH36366, Vol. 3, p. 1848 (1999).

16. T. F. Wang, Phys. Rev. E 61, 855 (2000).

17. See, for example, Sec. 4.2 in Global Bifurcations and Chaos: Analytical Methods, by S. Wiggins (Springer-Verlag, New York, 1988), for discussions on multi-frequency parametrically forced systems.

18. R. L. Gluckstern, W.-H. Cheng, and H. Ye, Phys. Rev. Lett. 75, 2835 (1995).

19. I. M. Kapchinskij and V. V. Vladimirskij. Proc. of the 2nd. Intl. Conf. on High Energy Accelerators, p. 274 (CERN, Geneva, 1959). 